\title{
Experimental identification of the dominant fabric in widely graded soils
}

\author{
H. Jentsch, P. Winkler, M. R. Salehi Sadaghiani, K.J. Witt \\ Bauhaus-Universität Weimar, Department of Geotechnical Engineering, Weimar, Germany
}

\begin{abstract}
To estimate the vulnerability of widely graded soils to internal erosion, the soil structure has to be analysed. Based on the theory of the bimodal fabric, each particle size distribution (PSD) has a unique diameter that divides the PSD into particles of the soil skeleton and finer mobile particles. The particle diameter, which divides the PSD into these two fractions, is defined as the separation point $\left(\mathrm{d}_{\mathrm{T}}\right)$ and part of the soil skeleton. In this study the Sequential Fill Test (SFT) is used to identify the mobility of particle fractions and the soil matrix.

This study is an extension of a series of descriptions of this particular test method. The paper is focused on a widely graded PSD, which is prone to internal erosion. This study demonstrates the change of the soil matrix by variation of the amount of the fine particles. The soil matrix of the investigated PSD does not change until the pore volume is completely filled with mobile particles. If the mobile particles filled completely the pore volume of the coarse soil skeleton, they become also part of the soil skeleton. This matrix is called dual matrix. With further increase of the amount of fine particles, the coarse skeleton is replaced by a new skeleton, where all particles belong to the soil skeleton.

For the assessment of a selected PSD, which is prone to internal erosion, it is important to identify the amount of fine fractions, which can stabilize an internally instable dominant fine matrix (i.e. building a dual matrix).
\end{abstract}

\section{INTRODUCTION}

The choice of materials and construction conditions is an important aspect for the safety of embankment dams. Often the local available soils have to be used for the construction of embankments. These are often non-cohesive sediments and widely graded soils with different amounts of fine fractions. Due to their widely graded characteristic, these soils are prone to suffusion. The transport of fine particles is a complex and iterative process, which is directly connected with the mobility of a single particle and the pore paths of the soil skeleton. Therefore the identification of the mobile fine particles has an important influence on the assessment of soils stability.

Finer soil particles, which are smaller than the minimum diameter of a pore path, can be washed out of the soil skeleton by seepage forces. Soils prone to suffusion are described as internally unstable (Kenny \& Lau 1986). The challenge in assessing the internally unstable soils to estimate the soil structure or the effective size of mobile particles (Sadaghiani \& Witt 2011). According to recent studies the soil can be considered as a bimodal structure, consisting of soil skeleton and fill (Witt, 2013). The coarser particles are assumed to have a fixed posi- tion and form the skeleton. Soil transmits the stresses through its skeleton. Potentially mobile particles are embedded into the pores of the soil skeleton (Kézdi, 1979; Kenny \&Lau, 1986; Burenkova, 1993, Wan \& Fell, 2008; Witt, 2013).

For assessment of the internal stability of a particle size distribution (PSD), it is not only important to characterize the soil as internally stable or unstable, but also to evaluate the amount of potentially erodible particles. For quantification of those erodible particles, the potentially mobile and the skeletonforming particles must be identified. Theoretically, the fractions which belong to the fill can be increased till the pore space is totally filled. From that point, by further increase of the fine fraction, they will behave more and more as skeleton. For the structure analysis of the PSD, it is important to identify the amount of the added fractions, which can shift the separation point to smaller diameters.

The aim of this paper is to identify the structure (matrix) of a granular packing, based of experimental investigations. Furthermore, the structural change by different amounts of fine fractions was investigated. 


\section{THEORY OF GRANULAR PACKING}

For the classification of granular packings a theoretical model was developed (see Figure 1). The model describes characteristic packings and there changes of structure during a successive addition of fine particles. Here, the packing is observed from the mesolevel. All particles are idealized as spheres. A homogeneous distribution of the particles and the densest possible packing is assumed

A highly poly-disperse packing is formed in this model by two different particle clusters. The particles of the skeleton, which transfer the stress of a mechanical loads, and the mobile particles which are loosely embedded in the pore space of the static skeleton. The loose or mobile particles can be washed-out or filter along the pore paths which are formed by the structure of the packing. Depending on the particle size distribution different granular structures (matrix) are formed. This structures need to be identified and characterised.

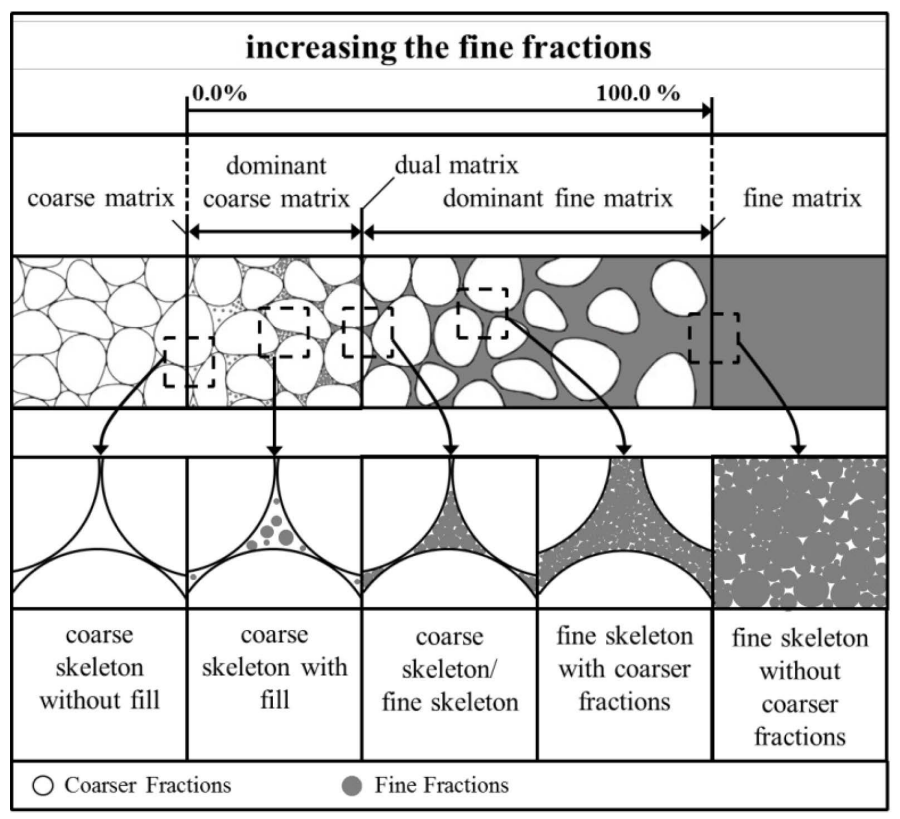

Figure 1. Categories of structure for granular packings.

The theoretical model of packings consists of five basic matrix types. Starting from a coarse matrix in which all particles form the skeleton, fine particle have gradually increased. By increasing the percentage of fine particles the structure changes into a dominant coarse matrix. In this structure the skeleton is mostly formed by the coarse fractions. It should be noted that a small percentage of fine particles also belongs to the skeleton.

The fine fractions embedded in the pore spaces, do not transmit stress and therefore are mobile. A transport of this fine particles due to a hydraulic load is possible. If the fine particles further increased, the pore space is filled with more mobile particles. Once the pore space has reached a $100 \%$ filling with fine particles, the structure shifts to a dual matrix. The fi- ne fractions form a secondary fine skeleton in the pore space. In this stage fine and coarse particles transmit stresses. Furthermore the fill effect causes a refinement of the pore paths. An example for such a case is the Fuller curve (Fuller-Thompson. 1907). With a further increase of the fine fractions, the coarse skeleton dissolves. The coarse fractions are now embedded in the fine particles and all particles are forming the skeleton. This matrix is defined as dominant fine matrix. By an amount of $100 \%$ fine particles in the packing only the fine particles form the skeleton. Neglecting the scale, the fine matrix has the same characteristics as the coarse matrix.

\section{METHODS OF IDENTIFICATION}

Based on the presented theoretical matrix types several experimental methods of identification have been developed and at the Bauhaus-Universität Weimar. With these laboratory tests the presented matrix types and the kinematic of the mobile particles can be identified and analysed. In this paper the Sequential Fill Test (SFT) is used to identify the matrix type.

\subsection{Sequential Fill Test}

Burenkova (1993) has developed a test procedure, in which the soil was sieved and divided into its fractions. First, the coarsest fraction $\left(\mathrm{d}_{\max }\right)$ was built into a test cylinder. After that, the next finer fraction was added and the new PSD built again into the test cylinder (Figure 2). In each step the volume of the soil

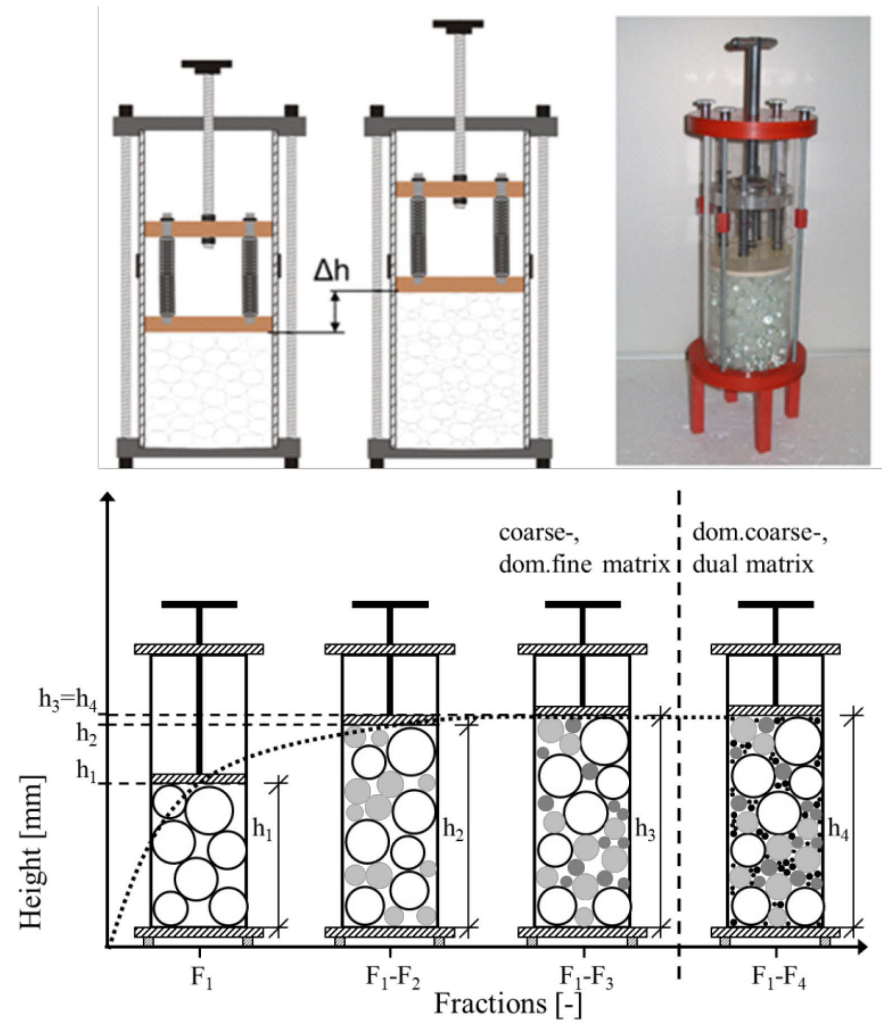

Figure 2. A view of the Sequential Fill Test (SFT) and the measurement procedure. (Salehi Sadaghiani et al. 2012) 
was measured. The test is finished when all fractions are added into the test cylinder. If the volume of the soil in each step is increased, the fractions are considered to belong to the soil skeleton. There is no published image or illustration of the Burenkova's test device (Burenkova 1993).

Binner et al. (2010) has shown for the first time a simple apparatus, in which the SFT was accomplished. The apparatus was designed by the Federal Institute for Materials Research and Testing (BAM) for identification of granular soil structures. The same apparatus was adapted and used for measurements at Bauhaus-Universität Weimar. A schematic diagram of the apparatus for the tests is given in Figure 2. The measurement values are the volume and the porosity of the packing.

The aim of this experiment is to determine the particle size fractions which forms the skeleton in a soil. The experiments were performed with assumption that the particle fractions, which do not belong to the skeleton, will not change the height of soil column (see Eq.1, Eq.2).

This fractions are equal to the mobile fine fill particles in section 2. It is useful to describe the test results as a relative height in percent. If the height of sample consisted of certain number of fractions is $h_{i}$, and the biggest height of the sample which is built in the test device $h_{\max }$, the relative height $h_{r}$ in percent is defined by the formula:

$$
\begin{aligned}
& \mathrm{h}_{\mathrm{r}}=\mathrm{h}_{\mathrm{i}} / \mathrm{h}_{\max } \\
& \mathrm{h}_{\mathrm{i}}=\mathrm{h}_{\max } \quad \rightarrow \mathrm{d}_{\mathrm{T}}
\end{aligned}
$$

The diameter which divides the PSD into the skeleton and the loose fill fractions is defined as the separation diameter $\left(\mathrm{d}_{\mathrm{T}}\right)$ and belongs to the skeleton of the soil. Depending on the separation diameter the mobility of every fraction can be classified.

It should be noted here, that the SFT gives only a classification of the mobility for the whole fraction. It is not possible to characterise the mobility for a single particle. However this only applies to the mobile fine particles and a very small percentage of the fraction of the separation diameter. For example in a granular packing with a high percentage of fine fractions, it is not clear which particles belong to the skeleton or mobile particles. This is caused by the high number of fine particles, which increases the number of stable combinations of particles. This has minor effect on the results, because the assessment of the mobility is always performed for an entire fraction.

The classification of a fraction can be defined in dependency of the separation diameter as follows:

$\mathrm{d}_{\mathrm{i}} \geq \mathrm{d}_{\mathrm{T}} \rightarrow$ fractions belongs to the skeleton

$\mathrm{d}_{\mathrm{i}}<\mathrm{d}_{\mathrm{T}} \rightarrow$ fraction belongs to the mobile particles

\subsection{Materials}

The investigated PSD presents a non-cohesive, widely graded soil (see Figure 3). This Soil is considered as prone to suffusion, e.g. after Wan \& Fell (2008) and Witt (2013). The soil is typical for sediments in the medium course of a large river. The original material was taken from a fluviatile soil of the Upper-Rhine river area.

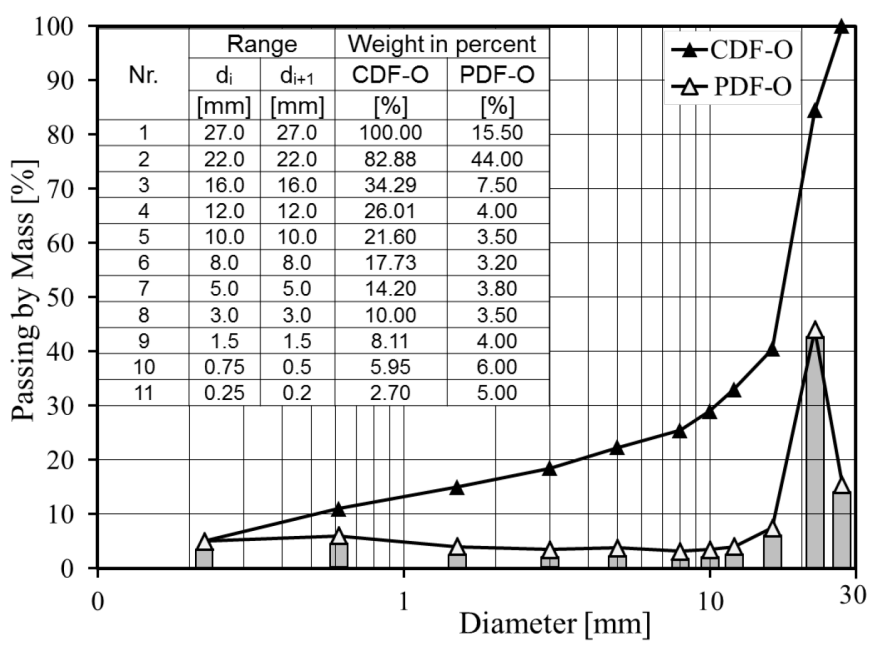

CDF-O. : Cumulative Distribution Function-Original PSD

PDF-O. : Probability Distribution Function-Original

Figure 3. Investigated particle size distribution.

This PSD is used for the experimental identification of the dominant fabric. Therefore it was approximated with 11 Fractions. The soils particles have been replaced with glass beads for all tests. They offer the advantage of less variation in shape and surface friction. Moreover, it has been proved that with glass beads detecting anomalies in the experiments is easier than working with soil (Salehi Sadaghiani et al. 2012). The particles of each fractions from 1 to 9 have a constant diameter. Fraction $10(0.75-0.5$ $\mathrm{mm})$ and $11(0.25-0.2 \mathrm{~mm})$ have uniform distribution, which is shown in the Figure 3 with an average diameter value.

\subsection{Experimental Testing Strategy}

According to the defined objectives set in section 1, the skeleton-forming and the mobile particles were determined by using the described method of identification (SFT). Based on the results the matrix is derived. Afterwards the change in the matrix type (section 2) is simulated by varying the mobile fine particles. Based on the theoretical model of granular packings, the dual matrix can be identified by measuring the porosity. With an increase of the fine particles the pore space has been filled completely with fine particles (dual matrix, Figure 1) and the porosity reaches a minimum. 


\section{EXPERIMENTS}

\subsection{SFT - Original Soil}

For predicting the dominant matrix in the soil structure of the chosen PSD the proposed methods were applied. Figure 4 shows the results of the SFT for the original PSD, in which the separation point of $\mathrm{d}_{\mathrm{T}}=3 \mathrm{~mm}$ can be seen. The skeleton consists of fractions from $27 \mathrm{~mm}$ to $3 \mathrm{~mm}$. All other fine fractions belong to the fill $(1.5-0.2 \mathrm{~mm})$.

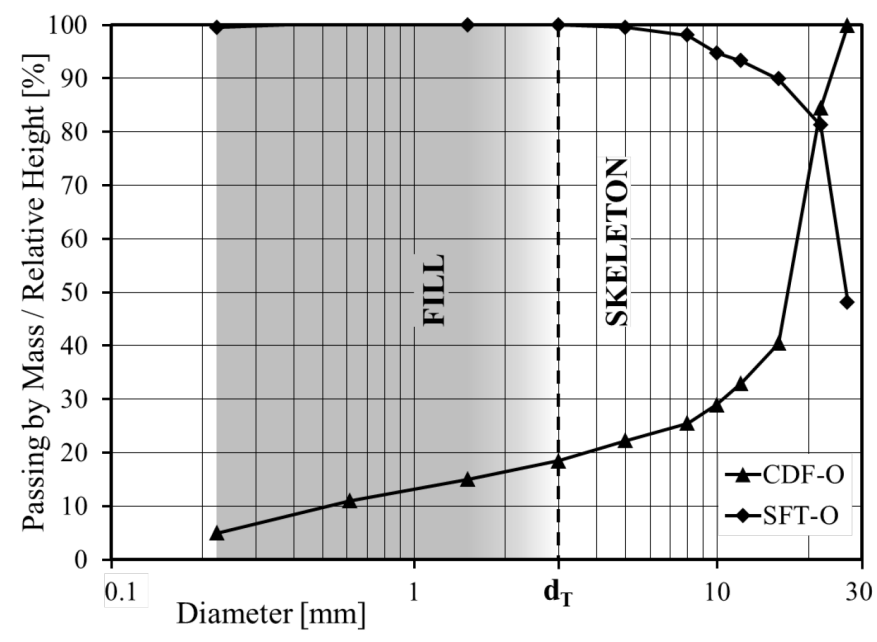

CDF-O. : Cumulative Distribution Function Original Soil PSD

SFT-O. : Sequential Fill Test-Original Soil (Relative Height)

$\mathrm{d}_{\mathrm{T}} \quad$ : Separation Point

Figure 4. Results of SFT for original PSD

\subsection{Variation of Fine Particles}

For the simulation of the change in structure the amount of fine particles was linear increased gradually. For the variation of the PSD see Figure 5.

According to section 2 and 3.1 a low number of the particles of the fraction of the separation diameter is mobile. This can be illustrated using a model of spheres. Based on the model of the densest sphere packing (possible combinations of 4 spheres) it can be shown that, for some combinations a stable system doesn't exist (e.g. diameters: 27, 27,12 and 3). In that special case the diameter is a mobile particle. From the calculation of the constriction size distribution (Silveira, 1965) there are also combinations known, which constriction sizes are bigger than the separation diameter (e.g. diameters: 27, 22, 16 $\mathrm{d}_{\mathrm{CSD}}=3.22$ ). Therefore, the mobile particle can be transported through the pore paths and so the fraction of the separation diameter has to be included in the variation of the fine fractions.

For an exact determination of the change in the structure, each mobile fraction must be varied separately. In this study the variation of a separate fraction was not carried out, to keep the experiment clear. Figure 5 shows the variation of the fine fractions.

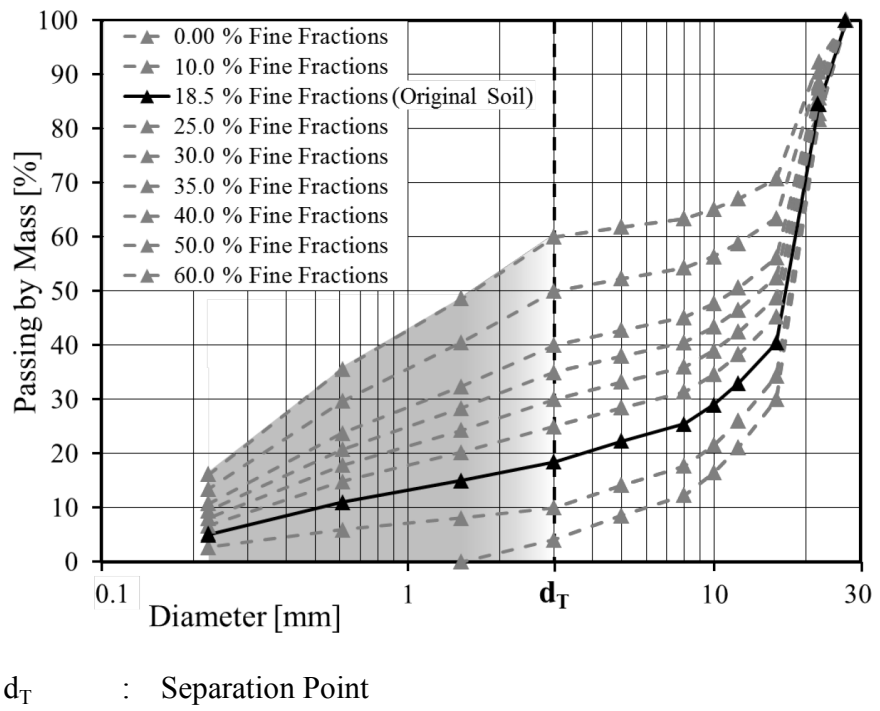

Figure 5. Variation of the original PSD

\section{RESULTS}

The test was performed with a constant mass $(6.0 \mathrm{~kg})$ for all investigated PSDs. Thus, the volume of the PSDs and the related porosity can be determined directly from the test. The porosity is measured by the necessary volume of water, which is needed to fill the pore space completely.

From Figure 6 it can be seen, that the porosity decreases in a range of $0.0 \%$ to $35.0 \%$-mass of fine fraction. The fine particles are embedded into the pore space and reduce the pore volume. This range $(0.0 \% \leq$ fine fractions $<35.0 \%)$ represents a dominant coarse matrix and is internal unstable.

At an amount of $35.0 \%$ fine fraction, a minimal porosity is measured. At that point the pore space is completely filled with fine particles and the mobile fractions transformed to skeleton-forming particles. This transition is defined as a dual matrix and stable against internal erosion.

If the amount of fine fractions is exceeded $35.0 \%$, the coarse skeleton is replaced by a fine skeleton. The low number of the large pores of the coarse skeleton changed into a high number of small pores of the fine skeleton. The porosity increases and an internal stable dominant fine matrix establishes.

\section{VALIDATION WITH GEOMETRICAL CRITERIA}

The validation of the experimental results of the method of identification (section 3 and 5) was analysed comparatively with different criteria against internal erosion (e.g. Kenny \& Lau, 1986; Burenkova, 1993; Wan \& Fell, 2008; Witt, 2013). The analyses was carried out using the method of Wan \& Fell (2008) and Witt (2013). 


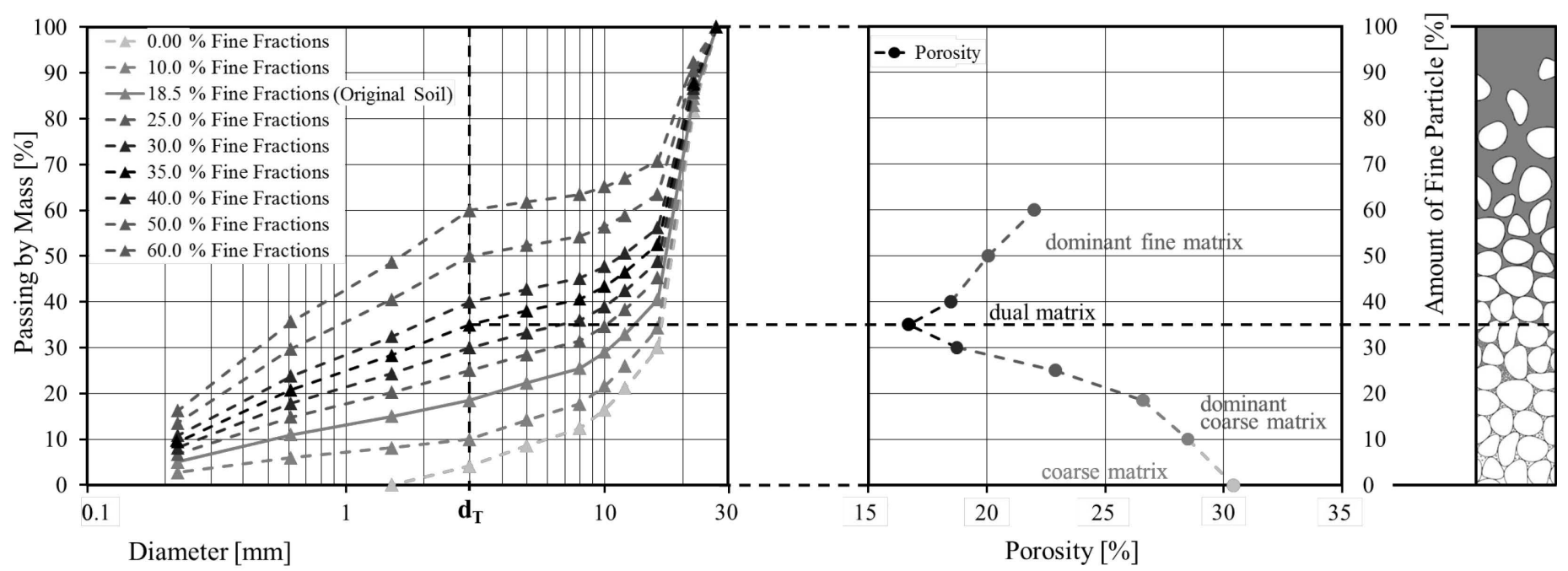

Figure 6. Results-Variation of the original PSD

The method of Witt (2013) is further development of Kenny \& Lau's method. The slope is calculated here for a constant distance between two particle diameters $(\mathrm{d} \rightarrow 4 \mathrm{~d})$. The slope is called as selffiltration index $\left(\mathrm{I}_{\mathrm{SF}}\right)$ and characterizes the retention capacity of the soil skeleton towards mobile fines fractions. This method is based on theoretical analysis and experimental investigations. The analysis was based on a $\mathrm{I}_{\mathrm{SF}} \leq 9$. This admits material transport, but prevents a global material discharge.

The method of Wan \& Fell (2008) is based on experimental investigation. There stability criterion depended on the slope of the coarse fractions $\left(\mathrm{d}_{90} / \mathrm{d}_{60}\right)$ and the fine fractions $\left(\mathrm{d}_{20} / \mathrm{d}_{5}\right)$. From this criterion, different zones of the PSD are derived, from which the internal erosion can be assessed. They added a transition zone to the unstable and stable zone. The subdivision is based on a experimentally determined likelihood of internal instability. Between the zones are sharp boundaries.

Table 1. Comparison of the Test Results with geometric criteria

\begin{tabular}{rlll}
\hline \multicolumn{1}{c}{ FF } & $\begin{array}{c}\text { Wan \& Fell } \\
(2008)\end{array}$ & $\begin{array}{c}\text { Witt } \\
(2013)\end{array}$ & Test Results \\
\hline 0.0 & stable & stable & coarse matrix \\
10.0 & unstable & unstable & dom. coarse matrix \\
18.5 & unstable $^{1}$ & unstable & dom. coarse matrix \\
25.0 & un-/stable $^{2}$ & unstable & dom. coarse matrix \\
30.0 & un-/stable $^{2}$ & unstable & dom. coarse matrix \\
35.0 & stable & stable & dual matrix \\
40.0 & stable & stable & dom. fine matrix \\
50.0 & stable & stable & dom. fine matrix \\
60.0 & stable & stable & dom. fine matrix \\
\hline
\end{tabular}

1) transition zone

2) closed to the boundary, stable zone/transition zone $(22.9>22)$

The comparison of the experimental results with the analytically calculated stability against internal erosion shows a good correlation (see Table 1). The analytical results differ only at the transition from dominant coarse matrix to dual matrix. The experimental determined transition at 35.0\%-mass fine fractions could be reproduced with the method from Witt (2013). With the method from Wan \& Fell (2008) the transition has been predicted at 25.0$30.0 \%$ fine fractions. However, the results for an amount of fine fractions of $25.0-30.0 \%$ are located in the transition zone or closed to the boundary condition between stable and transition zone. Despite these small differences, the experimental results of the proposed identification method could be approximately reproduced by the analytical methods (Witt, Wan \& Fell), which are also based on experimental studies.

\section{CONCLUSION}

To assess the internal stability of a soil, the most important aspect is the estimation of its structure. The soil structure can be hypothetically assumed as a bimodal structure, consisting of fixed soil skeleton and fill (Witt 2013). The main objective of this study is to identify the soil structure of a PSD, investigate the percentage of fine fractions in a packing and its influence on the soil structure. Therefore, a experimental method for determination of dominant soil matrix is proposed. The behaviour of the structure by addition of fine fractions is investigated to get an internally stable soil structure.

In this study it is shown, if the amount of the fill fraction is increased the pore spaces can be totally filled with them, so that a shift between different matrixes occur. Here of particular interest is the transition between the dominant coarse matrix and the dominant fine matrix, which is called dual matrix. The dual matrix characterises the transition of the mobile particles to skeleton-forming particles (here 35.0\% amount of fine fractions, see Fig. 6), which are firmly integrated in the soil skeleton and can transmit stresses. The results also agree with analytical calculations (Witt, 2013; Wan \& Fell, 2008). 


\section{REFERENCES}

Binner R., Homberg U., Prohaska S., Kalbe U., Witt K.J., 2010: Identification of descriptive parameters of the soil pore structure using experiments and CT data, Proc. 5th. Int. Conf. Scour Erosion (ICSE-5), pp. 397-407.

Burenkova V.V., 1993: Assessment of suffusion in noncohesive and graded soils, Proc. 1st. Int. Conf. GeoFilters, Karlsruhe, Germany, Balkema, Rotherdam, The Netherlands, pp. 357-360.

Fuller W.B., Thompson E., 1907: The laws of proportioning concrete, ASCE Transactions, Nr. LIX: pp. 67-118.

Kenny T. C. \& Lau, D., 1986: Internal stability of granular filters, Reply, Canadian Geotechnical Journal, 23, pp.420423.

Kézdi Á., 1979: Soil Physics; selected topics, In: K. Á. (ed.): Developments in Geotechnical Engineering, Elsevier Amsterdam.

Salehi Sadaghiani M. R. \& Witt K. J., 2011: Experimental identification of mobile particles in suffusible non cohesive soils, European Journal of Environmental and Civil Engineering, 15, pp. 1155-1165.

Salehi Sadaghiani M. R., Witt K.J., Jentsch H., 2012: A Statistical Approach to Identify Mobile Particles of Widely Graded Soils, in 6th International Conference of Scour and Erosion, Paris, pp. 839-845.

Silveira A.F.,1965: An Analysis of the Problem of Washing Through in Protective Filters, in 6th Int. Conf. on Soil Mechanics and Foundation Engineering Bd. 2, Montreal, pp. 551-555.

Wan C. \& Fell R., 2008: Assessing the Potential of Internal Instability and Suffusion in Embankment Dams and Their Foundations, Journal of Geotechnical and Geoenvironmental Engineering, 134, pp. 401-407.

Witt K.J., 2013: Das Selbstfiltrationsindex als Suffosionskriterium für nichtbindige Erdstoffe, Geotechnik, 8. 